\title{
Understanding charge transfer processes on metal oxides - A Laser-Flash-Photolysis study
}

\author{
Fabian Sieland*a, Jenny Schneider ${ }^{\mathrm{a}}$, Thorsten Lippmann ${ }^{\mathrm{a}}$, Detlef W. Bahnemann ${ }^{\mathrm{a}, \mathrm{b}}$ \\ ${ }^{a}$ Institute of Technical Chemistry, Leibniz University Hannover, Callinstr. 3, 30167 Hannover, \\ Germany; 'Laboratory "Photoactive Nanocomposite Materials", Saint-Petersburg State University, \\ Ulyanovskaya Str. 1, Peterhof, Saint-Petersburg, 198504, Russia
}

\begin{abstract}
In the focus of this study, mixtures of commercially available $\mathrm{TiO}_{2}$ powders were created and their photocatalytic activity concerning the acetaldehyde degradation in the gas phase was tested. Further, the lifetime of the photogenerated charge carriers was analyzed by Laser-Flash-Photolysis-Spectroscopy. The acetaldehyde degradation experiments of the mixed powders lead to positive and negative deviations from the expected weighted mean. Nevertheless, their photocatalytic activity could be correlated with the lifetime of the charge carriers. A longer charge carrier lifetime at ambient conditions correlated with a lower fractional conversion of acetaldehyde. The advantageous activities of the samples were associated with a charge transfer reaction between larger and smaller particles comparable to the antenna mechanism. ${ }^{1}$

Keywords: Laser-Flash-Photolysis-Spectroscopy, charge transfer, semiconductor, photocatalysis, kinetics, acetaldehyde degradation
\end{abstract}

\section{INTRODUCTION}

In the area of photocatalysis many multistep reactions are judged and compared according to their overall yield, e.g., determining the photonic efficiency. In many cases effects of the physical properties of the catalyst, such as morphology, crystallinity or BET-surface area, are detected empirically, however, the reason for advantages or disadvantages remains unknown. Laser-Flash-Photolysis-Spectroscopy offers the quantitative and time resolved detection of charge carriers in the material, which allows the analysis of the essential elementary steps of the photocatalytic reaction. Moreover, improvement of photocatalytic processes is only possible if the rate-limiting step can be determined.

Recent research indicates that charge carrier lifetimes in $\mathrm{TiO}_{2}$ depend on the particle size. ${ }^{2}$ Smaller $\mathrm{TiO}_{2}$ particles show shorter lifetimes than larger ones. A possible explanation is the negative effect of surface recombination. ${ }^{3}$ Nevertheless, a large interface between the catalyst and the environment is required to adsorb reactants and to carry out the charge transfer reaction. Clearly visible is the beneficial effect of a higher surface area for reactions with direct hole transfer, for these reactions the photocatalytic activity increases with increasing surface area. ${ }^{4}$ Essentially, the knowledge about different charge carrier lifetimes should enable the development of a photocatalyst with higher photocatalytic activity. A mixture of $\mathrm{TiO}_{2}$ with binary particle size distribution might combine the advantages of smaller and larger particles.

\subsection{Charge transfer between $\mathrm{TiO}_{2}$ particles}

In 2006 a synergetic mechanism of the charge transfer between $\mathrm{TiO}_{2}$ particles was suggested by Wang et al.. ${ }^{1}$ According to this concept the three dimensional structure of aggregated $\mathrm{TiO}_{2}$ nanoparticles in solution is able to transfer charge carriers throughout the network. This makes it possible that light absorption and the corresponding charge carrier generation takes place at a different particle than the charge transfer or the degradation of adsorbed molecules. This concept is named antenna mechanism and is illustrated in Figure 1. The present study aims to find evidence for the proposed charge transfer process. If such a transfer of electrons or holes between different particles is not possible, mixing of $\mathrm{TiO}_{2}$ powders should only lead to the weighted mean of the photocatalytic activities. In this case each particle would contribute separately to the overall quantum efficiency. A positive or negative deviation of this behavior would be a strong indicator for a charge transfer mechanism between particles, which might enable the development of photocatalysts with higher photocatalytic activity.

*sieland@iftc.uni-hannover.de; phone 49511 762-16043; fax 49511 762-2774; tci.uni-hannover.de

Solar Hydrogen and Nanotechnology XI, edited by Chung-Li Dong, Proc. of SPIE Vol. 9935, 99350G · (c) 2016 SPIE · CCC code: 0277-786X/16/\$18 · doi: 10.1117/12.2239261 


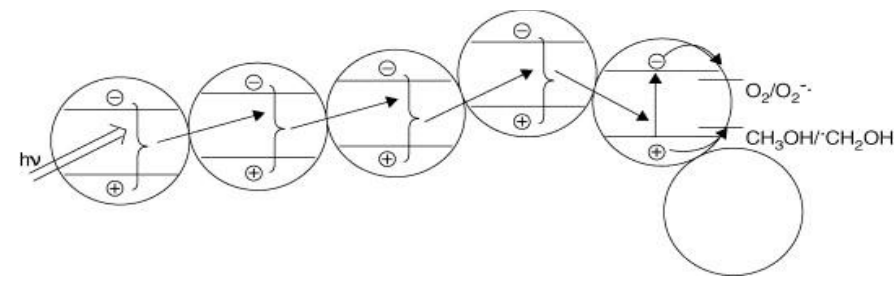

Figure 1. Cooperative charge transfer mechanism in three dimensional $\mathrm{TiO}_{2}$ networks - Antenna mechanism. ${ }^{1}$

\subsection{Laser-Flash-Photolysis-Spectroscopy}

The principle of laser excitation for the detection of charge carriers in optically dense materials was developed by the group of Wilkinson in 1981. ${ }^{5}$ The time-resolved measurement showed great potential for the analysis of excited states and photocatalytic reactions. The simple process of short time laser excitation accompanied by fast spectroscopy remained unchanged until today; the schematic set-up is shown in Figure 2.

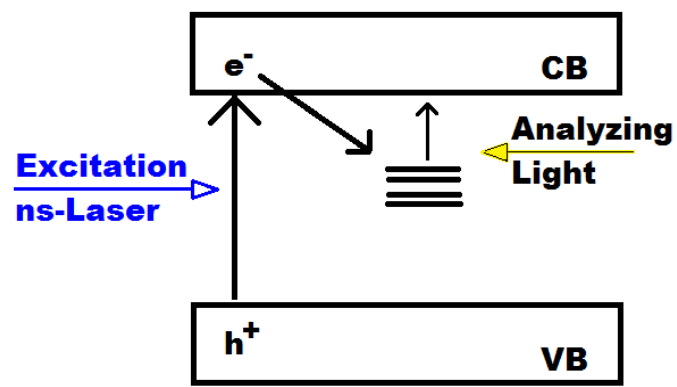

Figure 2. Schematic diagram of the analysis of a semiconductor by Laser-Flash-Photolysis-Spectroscopy.

In general, the Laser-Flash-Photolysis-Spectroscopy is a feasible tool for the quantitative detection of charge carriers in the nanosecond time scale. With the use of diffuse reflected instead of transmitted light the analysis of heterogeneous as well as homogeneous systems is possible. Charge carrier lifetimes can be observed from nano- to microseconds and can be correlated with the kinetics of charge transfer processes. In this study, Laser-Flash-Photolysis-Spectroscopy was used to examine the effects of particle size on charge carrier kinetics.

\section{EXPERIMENTAL}

\subsection{Materials}

All chemicals, which were used in this study, and the corresponding supplier and properties, are listed in Table 1. Commercial $\mathrm{TiO}_{2}$ powders from KRONOS International Inc. and Cristal with different particle sizes are named according to their BET surface area provided by the supplier $\left(\mathrm{TiO}_{2}-\mathrm{BET}\right)$.

Table 1. Materials

\begin{tabular}{|c|c|c|}
\hline Substance & Supplier & Properties \\
\hline $\mathrm{TiO}_{2}-350: \mathrm{PC} 500$ & Cristal & Anatase, $5-10 \mathrm{~nm}$ particles \\
\hline $\mathrm{TiO}_{2}-90:$ PC105 & Lyondell Chemical Comp. & $\begin{array}{l}\text { Anatase, } 15-25 \mathrm{~nm} \\
\text { particles }\end{array}$ \\
\hline $\mathrm{TiO}_{2}-12$ : Kronos 1001 & $\begin{array}{l}\text { KRONOS International, } \\
\text { Inc. }\end{array}$ & $\begin{array}{l}\text { Anatase, } 50-200 \mathrm{~nm} \\
\text { particles }\end{array}$ \\
\hline
\end{tabular}




\begin{tabular}{|l|l|l|}
\hline Substance & Supplier & Properties \\
\hline Acetaldehyde & The Linde Group & 217 ppm in Nitrogen \\
Water & EMD Millipore & Mill-Q System \\
Ethanol & Carl Roth GmbH + Co. & $>99,8 \%$ with 1\% \\
& KG & Methylethylketone \\
\hline
\end{tabular}

The surface area and particle size for the three different $\mathrm{TiO}_{2}$ powders are illustrated in Figure 3.

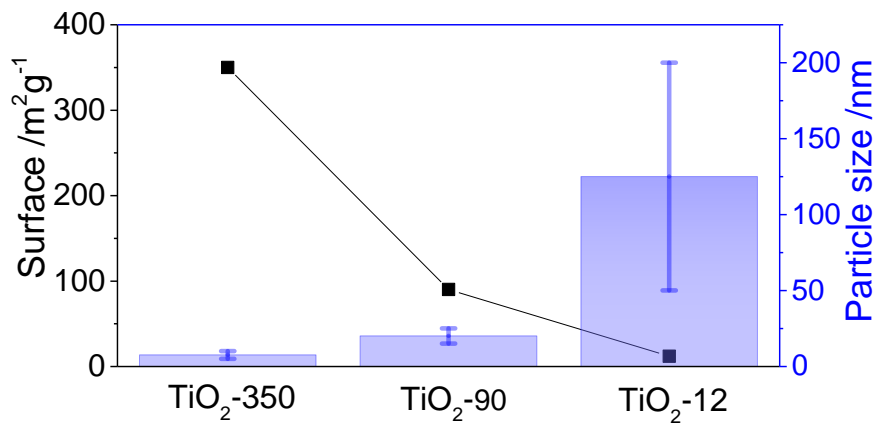

Figure 3. Surface area (black squares, left scale) and particle size (blue bars, right scale) of the used $\mathrm{TiO}_{2}$ basic materials.

\subsection{Preparation method of $\mathrm{TiO}_{2}$ mixtures}

The smaller particles of $\mathrm{TiO}_{2}-90$ or $\mathrm{TiO}_{2}-350$ were suspended in aqueous ethanol solution (10\% vol.- $\%$ ethanol) and treated with ultrasound $(340 \mathrm{~W} / \mathrm{L})$ for 5 minutes. During sonication the larger particles of $\mathrm{TiO}_{2}-12$ were added to reach a final concentration of $10 \mathrm{~g} / \mathrm{L} \mathrm{TiO}_{2}$. The ultrasound treatment was continued for further 3 minutes. The mixed suspension was centrifuged for 6 hours at $500 \mathrm{rpm}$. After sedimentation over night (10 hours) the supernatant was separated from the precipitate. Both fractions were dried at $70{ }^{\circ} \mathrm{C}$ until the combined weight reached the initial value. The reference material was treated accordingly.

Two series of new materials were synthesized by this process. The materials contained $0.4,5,20,40$, and $70 \mathrm{w}-\%$ of small particles $\mathrm{TiO}_{2}-90$ or $\mathrm{TiO}_{2}-350$. All results given in chapter 3. refer to these two series of powders.

\section{Variation of ultrasound treatment}

Prior to the above-described procedure, $\mathrm{TiO}_{2}-350, \mathrm{TiO}_{2}-12$ and $\mathrm{TiO}_{2}-12$ with $0.4 \mathrm{w}-\%$ of $\mathrm{TiO}_{2}-350$ were prepared with lower ultrasound energy densities ( $24 \mathrm{~W} / \mathrm{L})$. Those three samples were synthesized using 9 minutes of sonication as well. Further, two samples with $\mathrm{TiO}_{2}-12$ containing 5 and $20 \mathrm{w}-\%$ of $\mathrm{TiO}_{2}-350$ were prepared for 9 minutes using $340 \mathrm{~W} / \mathrm{L}$ ultrasound.

\subsection{Instrumentation}

Ultrasonic equipment: Branson 450 Digital Sonifier, 102-C Converter Centrifuge: Kendro Megafuge 1.0. Oven: Memmert $\mathrm{GmbH}+\mathrm{Co}$. KG BE 400. Acetaldehyde degradation was determined according to ISO 22197-2:2011 with the following equipment. Lamp: Philips HB 175 (4 x Philips Cleo 15 W, 365 nm). Flow meter: Brooks Instrument B.V. Smart Mass Flow 5850S. Gas chromatography: Synthech Spectras GC 955. TEM: FEI Tecnai G2 F20 TMP with 200 kV FEG. Laser Flash Photolysis Spectroscopy was done with Applied Photophysics LKS 80 Laser Flash Photolysis Spectrometer coupled with a Brilliant B Nd-YAG laser ( $3^{\text {rd }}$ harmonic, $\left.355 \mathrm{~nm}\right)$ from Quantel.

\subsection{Acetaldehyde degradation}

For the measurement of the acetaldehyde degradation according to ISO 22197-2, the synthesized powders were pestled and the received fine powder was pressed into the mould $(3.1 \times 3.1 \times 0.1 \mathrm{~cm})$ of the PTFE sample holder. Accordingly, the active surface was $9.6 \mathrm{~cm}^{2}$. Every sample was pretreated with $10 \mathrm{~W} / \mathrm{m}^{2} \mathrm{UV}$-light (365 nm, Philips Cleo) for 72 hours and stored in a vacuum tight desiccator prior to the experiment. The fractional conversion of $1 \mathrm{ppm}$ acetaldehyde in a gas 
flow of $1 \mathrm{~L} / \mathrm{min}$ synthetic air with $50 \%$ relative humidity under $10 \mathrm{~W} / \mathrm{m}^{2} \mathrm{UV}$ radiation $(365 \mathrm{~nm}$, Philips Cleo) was determined.

\subsection{Laser-Flash-Photolysis-Spectroscopy}

The change in reflectance of the powder surfaces in contact with air after 6 ns laser excitation with a $355 \mathrm{~nm}$ laser pulse were detected. Each pulse had the average energy of 5-6 mJ and irradiated an area of $1 \mathrm{~cm}^{2}$. The analyzing light from a xenon lamp was focused on the powder samples and the diffuse reflected light was collected with a lens and guided into the detector. The signals were detected between 700 and $400 \mathrm{~nm}$ (step size $20 \mathrm{~nm}$ ) and for every trace 12 measurements were averaged. Figure 4 shows a schematic diagram of the experimental set-up.

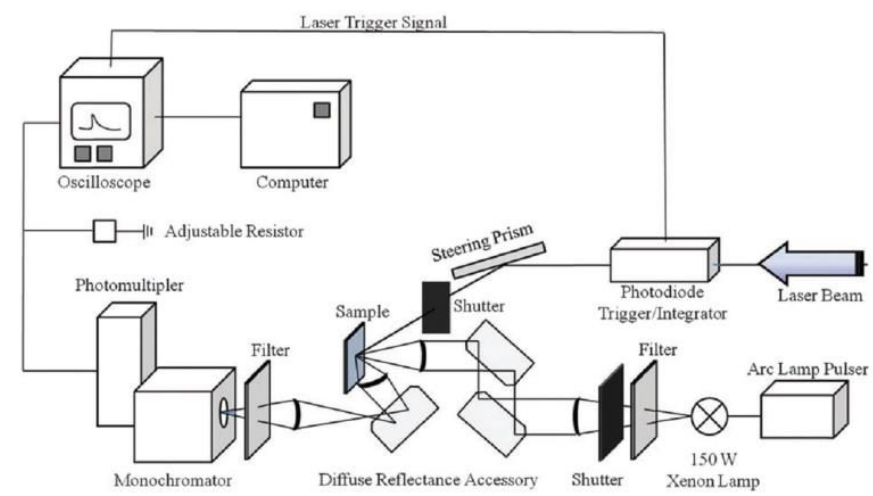

Figure 4. Scheme of the diffuse reflectance Laser-Flash-Photolysis set-up. ${ }^{6}$

\section{RESULTS}

\subsection{TEM images}

The TEM images shown in Figure 5 and 6 indicate a strong interaction between the different particles. The $\mathrm{small}_{\mathrm{TiO}_{2}-350}$ is distributed in small agglomerates on the surface of the larger $\mathrm{TiO}_{2}-12$ particles. Due to the ultrasound treatment nearly all of the small $\mathrm{TiO}_{2}-350$ is attached to $\mathrm{TiO}_{2}-12$ and no separated agglomerates of $\mathrm{TiO}_{2}-350$ are observed.

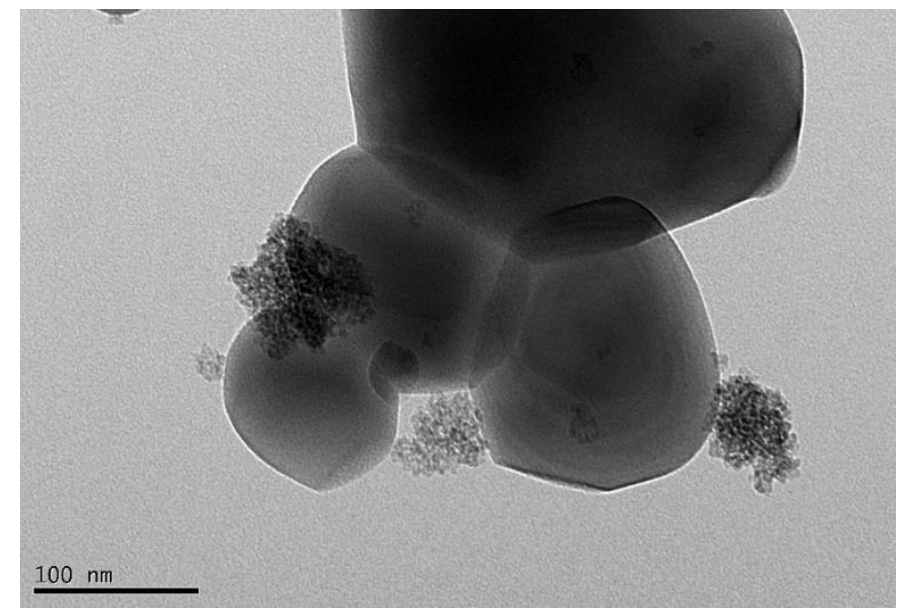

Figure 5. TEM image of $5 \%$ small $\mathrm{TiO}_{2}-350$ particles on large $\mathrm{TiO}_{2}-12$, synthesized via sonication ( $\left.340 \mathrm{~W} / \mathrm{L}\right)$ of aqueous ethanol suspensions of $\mathrm{TiO}_{2}$. 


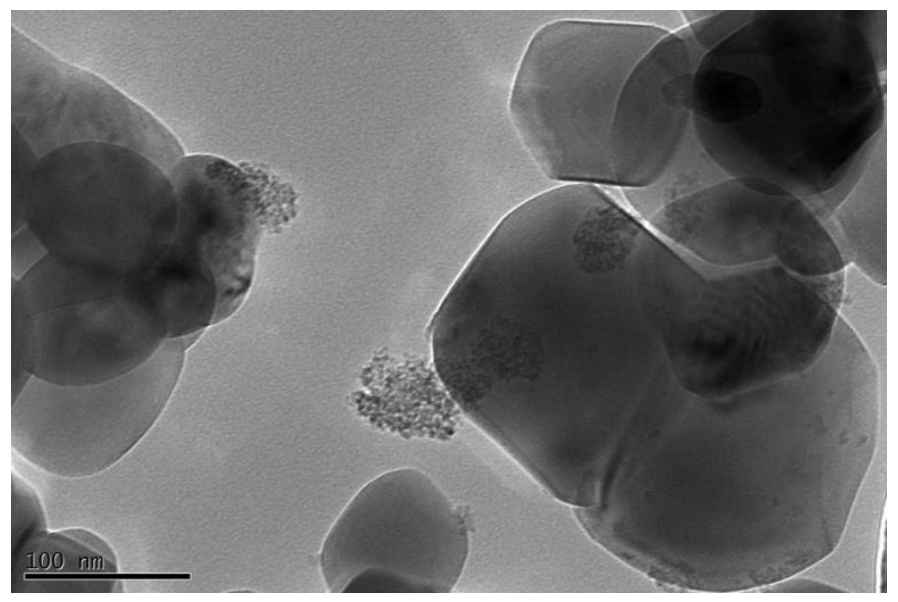

Figure 6. TEM image of $20 \%$ small $\mathrm{TiO}_{2}-350$ particles on large $\mathrm{TiO}_{2}-12$, synthesized via sonication (340 W/L) of aqueous ethanol suspensions of $\mathrm{TiO}_{2}$.

\subsection{Acetaldehyde degradation}

The photonic efficiencies were calculated from the results of the acetaldehyde degradation experiments.

$$
\xi=\frac{\Delta c \cdot \dot{V} \cdot p}{\Phi \cdot R \cdot T \cdot A}
$$

Equation (1) shows the correlation of the photonic efficiency $\xi$ and the experimental characteristics. The following notation is used: Fractional conversion of acetaldehyde $\Delta c$, volume flow $\dot{V}$, pressure $p$, photonic flux $\phi$, probing area of the catalyst A.

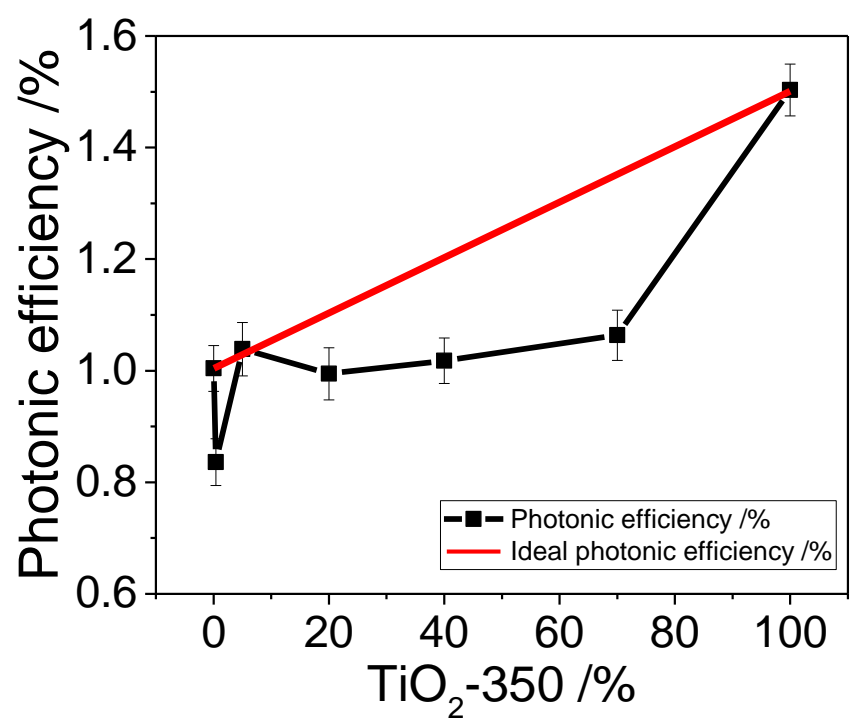

Figure 7. Photonic efficiencies of $\mathrm{TiO}_{2}-350$ mixed with $\mathrm{TiO}_{2}-12$ (black) and expected photonic efficiency of an ideal mixture (red).

For the mixtures between $\mathrm{TiO}_{2}-350$ and $\mathrm{TiO}_{2}-12$ particles, shown in Figure 7, we observed only negative impact on the photonic efficiencies. Very small additions of $0.4 \% \mathrm{TiO}_{2}-350$ by weight lead to a significant decrease of the acetaldehyde degradation. The result is contradictory to the expectations, since with $\mathrm{TiO}_{2}-350$ a material with higher surface area and 
higher photocatalytic activity is added. If no interaction between the particles takes place one would expect the behavior of an ideal mixture, which can be represented by the weighted mean (Eq. 2).

$$
\xi_{\text {ideal }}=\sum_{i} x_{i} \cdot \xi_{i}
$$

The photonic efficiencies for the behavior of ideal mixtures $\xi_{\text {ideal }}$ were calculated from the mass fraction of the components $x_{i}$ and are given in Figure 7 and 8 as red lines.

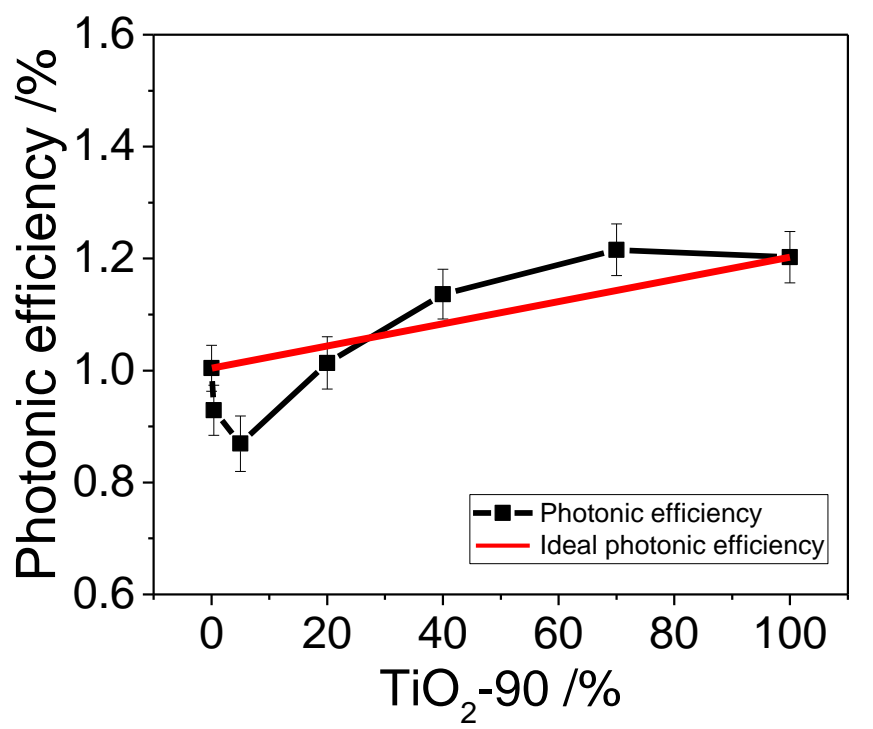

Figure 8. Photonic efficiencies of $\mathrm{TiO}_{2}-90$ mixed with $\mathrm{TiO}_{2}-12$ (black) and expected photonic efficiency of an ideal mixture (red).

Small amounts of $\mathrm{TiO}_{2}-90$ mixed with $\mathrm{TiO}_{2}-12$ displayed the same drop in photonic efficiency like mixtures with $\mathrm{TiO}_{2}-350$. Sample containing $40 \%$ or $70 \%$ of $\mathrm{TiO}_{2}-90$ had higher photonic efficiencies than expected. Moreover, the sample with $70 \%$ of $\mathrm{TiO}_{2}-90$ displayed the highest photonic efficiency, even slightly higher than the pure $\mathrm{TiO}_{2}-90$.

\subsection{Laser-Flash-Photolysis-Spectroscopy}

The optical change in reflectance at $400 \mathrm{~nm}$ of the powder surface was fitted with double exponential decay curves. The corresponding equation is shown below.

$$
y=y_{0}+A_{1} e^{-\alpha t}+A_{2} e^{-k t}
$$

The two fitted decay constants $\alpha$ and $k$ are listed in Table 2 for all tested samples. Until today, not all of the synthesized powder samples could be analyzed with Laser-Flash-Photolysis. Further research is planned and in progress.

Table 2. Kinetic fitting parameters Laser-Flash-Photolysis-Spectroscopy

\begin{tabular}{|l|l|l|}
\hline Sample & $\alpha / 10^{6} s^{-1}$ & $k / 10^{5} s^{-1}$ \\
\hline $\mathrm{TiO}_{2}-350$ & 140 & 15 \\
$\mathrm{TiO}_{2}-90$ & 5.1 & 7.4 \\
$\mathrm{TiO}_{2}-12$ & 6.4 & 4.4 \\
$5 \% \mathrm{TiO}_{2}-350$ & 8.1 & 6.2 \\
\hline
\end{tabular}




\begin{tabular}{|l|l|l|}
\hline Sample & $\alpha / 10^{6} s^{-1}$ & $k / 10^{5} s^{-1}$ \\
\hline $20 \% \mathrm{TiO}_{2}-350$ & 7.5 & 6.1 \\
$5 \% \mathrm{TiO}_{2}-90$ & 6.8 & 5.0 \\
$20 \% \mathrm{TiO}_{2}-90$ & 9.6 & 6.4 \\
\hline
\end{tabular}

To visualize the mathematic fitting process, the transient reflectance signal of $\mathrm{TiO}_{2}-12$ at $400 \mathrm{~nm}$ and the double exponential fit curve are shown in Figure 9.

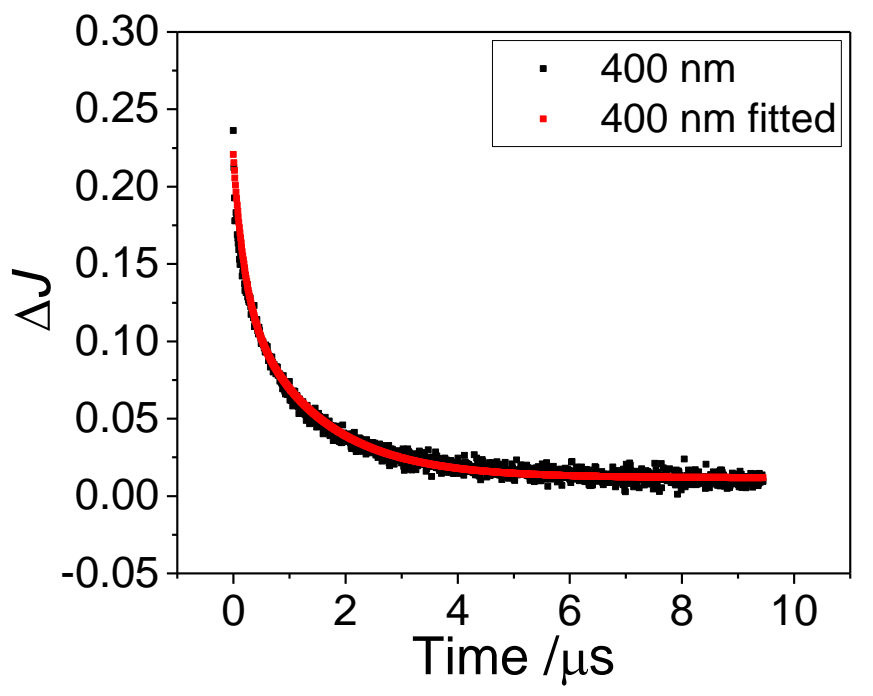

Figure 9. Transient reflectance signal at $400 \mathrm{~nm}$ of $\mathrm{TiO}_{2}-12$ (Average of 12 traces) after laser excitation with $5 \mathrm{~mJ} /$ pulse $\left(\lambda_{e x}=355 \mathrm{~nm}\right)$ (black) and double exponential fit (red).

\section{DISCUSSION}

In our prior experiments we used sonication with low energy density $(24 \mathrm{~W} / \mathrm{L})$ for mixing the powder samples. These experiments exhibited lower photonic efficiencies compared to those samples shown in chapter 3. Accordingly, a high energy density during sonication of the $\mathrm{TiO}_{2}$ suspensions proofed to be essential for achieving a good contact between the different particles. The ultrasound breaks up the agglomerated basic materials. Aiming for an intimate contact seemed necessary to reduce the energetic barrier the charge carriers have to overcome during charge transfer reactions. Since a longer ultrasound treatment with $340 \mathrm{~W} / \mathrm{L}$ energy density did not increase the photonic efficiency of the tested samples, we conclude that after $5 \mathrm{~min}$ of sonication we have a good contact between the different particles. The TEM images illustrate that small clusters of $\mathrm{TiO}_{2}-350$ particles can be generated on the surface of larger $\mathrm{TiO}_{2}$. This nano-structure should favor light absorption of the large $\mathrm{TiO}_{2}-12$ particles and, subsequently, charge transfer to smaller $\mathrm{TiO}_{2}$ where the large surface area facilitates the degradation of pollutants.

The results show that there is an effect of the binary particle size distribution on the photocatalytic activity of $\mathrm{TiO}_{2}$ powders. Unfortunately, addition of small amounts of small particles $\left(0.4 \mathrm{w}-\%\right.$ of $\mathrm{TiO}_{2}-350$ or $\left.\mathrm{TiO}_{2}-90\right)$ lead to a strong decrease of photonic efficiency (Fig. 7 and 8). This effect contradicts the expected behavior. Since, it is expected that the photonic efficiency increases, when a photocatalyst with higher surface area and higher acetaldehyde degradation is added. The reason might be an unfavorable charge transfer from the smaller to the large particles or a reduction of the available surface area due to the blocking of pores between the larger particles. However, for mixtures of $\mathrm{TiO}_{2}-90$ and $\mathrm{TiO}_{2}-12$ we could achieve an increased photonic efficiency, which can be attributed to a beneficial charge transfer mechanism between $\mathrm{TiO}_{2}$ particles. The proposed mechanism is displayed in Figure 10. 


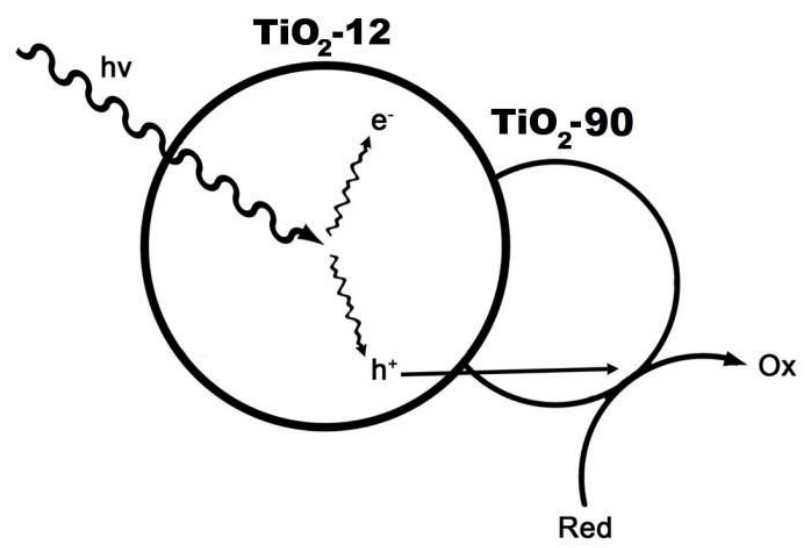

Figure 10. Synergetic charge transfer mechanism between $\mathrm{TiO}_{2}$ particles of different sizes.

The charge transfer of holes between small and large $\mathrm{TiO}_{2}$ particles might be the reason for the increased photooxidation of acetaldehyde on the surface. Since the smaller $\mathrm{TiO}_{2}-90$ particles have the higher surface area, which favors the adsorption of acetaldehyde, it is proposed that the oxidation is carried out at the surface of these particles. Hence, the transfer of holes to smaller particles takes place. This charge transfer can support the charge carrier separation and enhance the photocatalytic degradation reaction.

\subsection{Effect of surface area}

For the addition of larger amounts of small particles (40 and $70 \mathrm{w}-\%$ of $\mathrm{TiO}_{2}-350$ or $\left.\mathrm{TiO}_{2}-90\right)$ the effect on the photonic efficiency depends on the surface area of the particles. $\mathrm{TiO}_{2}-350$, with the highest surface area of $350 \mathrm{~m} / \mathrm{g}$, resulted only in a reduction of photonic efficiencies when it was mixed with $\mathrm{TiO}_{2}-12$. While the test series with $\mathrm{TiO}_{2}-90$ on $\mathrm{TiO}_{2}-12$ turned out to be beneficial for the photonic efficiency.

We have to take into account that pure $\mathrm{TiO}_{2}-350$ had the highest surface area and the highest acetaldehyde degradation in our experiments. It could be possible that the effect of surface area reduction due to the addition $\mathrm{TiO}_{2}-12$ was larger than the possible beneficial effect of charge carrier transfer. To support this theory it is recommended to run the following experiments: BET surface area and Laser-Flash-Photolysis-Spectroscopy of all samples. This work is under progress.

\subsection{Correlation of charge carrier kinetics and photocatalytic activity}

The exponential decay parameter $k$ of the signal at $400 \mathrm{~nm}$ describes the charge carrier lifetime. It describes the recombination kinetics of deeply trapped holes. ${ }^{7}$ Figure 11 and 12 compare the results of the acetaldehyde degradation with the exponential decay parameter $k$ for the basic materials and four mixtures with binary particle size distribution.

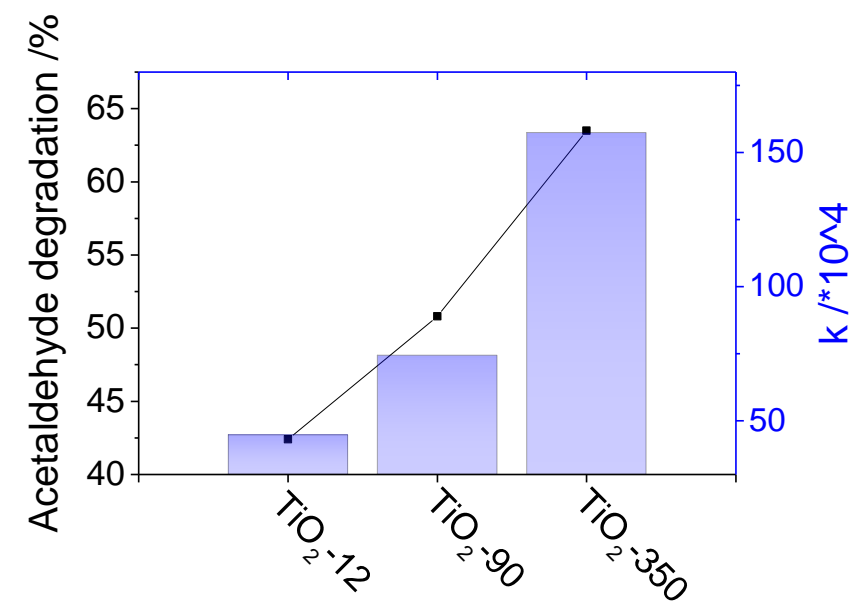

Figure 11. Acetaldehyde degradation (black) and exponential fit parameter $k$ (blue) for the basic materials. 


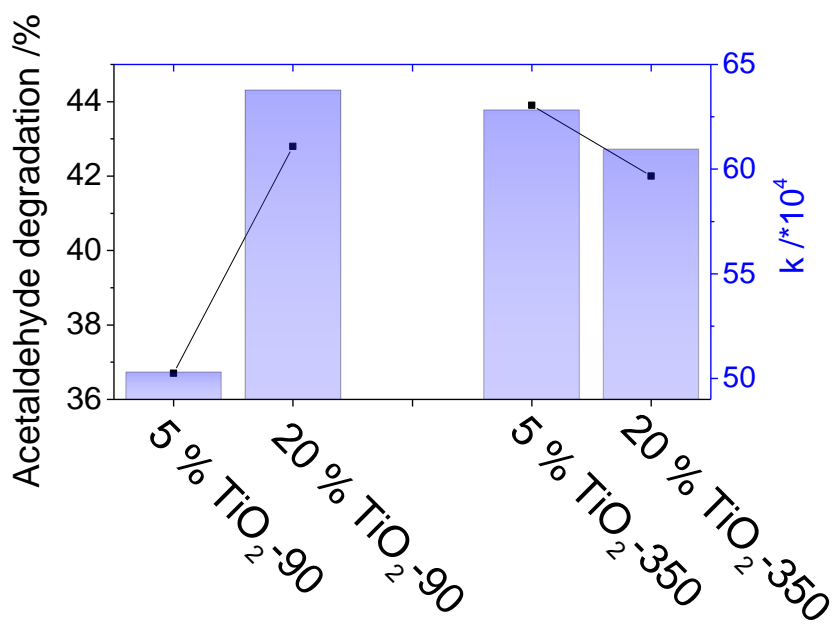

Figure 12. Acetaldehyde degradation (black) and exponential fit parameter $k$ (blue) for mixed $\mathrm{TiO}_{2} \mathrm{samples}$

It can be seen that the acetaldehyde degradation can easily be correlated with the exponential decay parameter $k$. Even for the three basic materials used in this study, the acetaldehyde degradation is higher for the materials exhibiting a higher parameter $k$. These results were supported from the acquired data for the synthesized mixtures of $\mathrm{TiO}_{2}-12 \mathrm{and} \mathrm{TiO}_{2}-350 / 90$. Similar to the correlation of the basic material we observed higher acetaldehyde degradation when raising the percentage of $\mathrm{TiO}_{2}-90$ from $5 \%$ to $10 \%$, the decay parameter grew accordingly. Interestingly, the acetaldehyde degradation could not be raised with the addition of $20 \% \mathrm{TiO}_{2}-350$, however, the correlation between the achieved acetaldehyde degradation result and the parameter $k$ was observed as well. We conclude that the reactivity towards acetaldehyde oxidation is proportional to the recombination kinetics of deeply trapped holes (Figure 13).

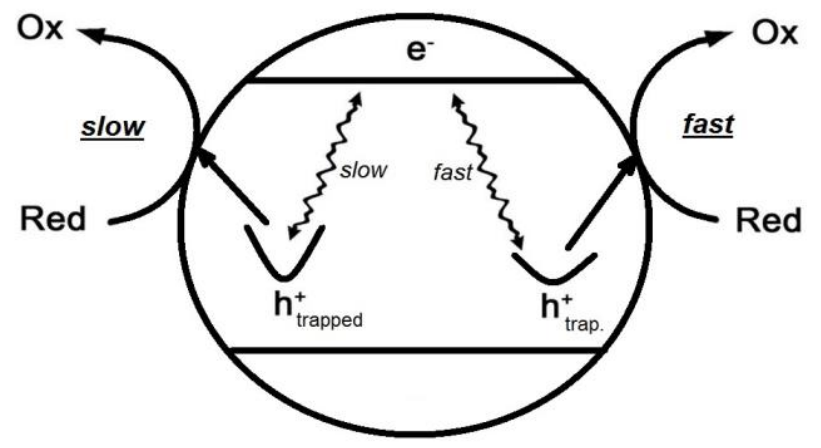

Figure 13. Correlation charge carrier recombination kinetics and degradation of adsorbed pollutants.

\section{CONCLUSION}

In the present study ultrasound treatment of $\mathrm{TiO}_{2}$ suspensions was used successfully to create mixtures with binary particle size distribution. TEM images proof that the smaller particles are attached to the surface of the larger particles and good contact between the different particles is achieved. The addition of $0.4 \mathrm{w}-\%$ of small $\mathrm{TiO}_{2}$ particles $\left(\mathrm{TiO}_{2}-350\right.$ and $\mathrm{TiO}_{2-}$ 90) to larger particles decreased the acetaldehyde degradation. This effect was similar for both kinds of small particles. Interestingly, for the mixtures containing more than $20 \%$ of the smaller particles the effects were opposite. The addition of $\mathrm{TiO}_{2}-350$ (surface area $350 \mathrm{~m}^{2} / \mathrm{g}$ ) strongly reduced the photocatalytic activity, while the addition of $\mathrm{TiO}_{2}-90($ surface area $90 \mathrm{~m}^{2} / \mathrm{g}$ ) turned out to be beneficial. The highest acetaldehyde degradation was achieved by a mixture of $70 \% \mathrm{TiO}_{2}-$ 
90 and $30 \% \mathrm{TiO}_{2}-12$. In sum, the experimental results support a synergistic charge transfer mechanism similar to the antenna mechanism. ${ }^{1}$

The Laser-Flash-Photolysis measurements indicate that the photonic efficiency of the acetaldehyde degradation is inversely proportional to the lifetime of trapped charge carriers. The charge carrier lifetime was evaluated from the exponential decay parameter $k$ of the transient change of reflectance at $400 \mathrm{~nm}$. In conclusion, our experimental results indicate a proportional correlation between recombination of deeply trapped holes and reactivity towards acetaldehyde oxidation.

\section{ACKNOWLEDGMENT}

We gratefully acknowledge the financial support from the Federal Ministry of Education and Research (BMBF) Project "PureBau" No. 13N13350. Furthermore, we thank the companies KRONOS International, Inc. and Cristal for providing the commercial $\mathrm{TiO}_{2}$ powders and the Laboratory of Nano and Quantum Engineering (LNQE) for the TEM measurements.

\section{REFERENCES}

[1] Wang, C., Pagel, R., Dohrmann, J. K.., Bahnemann, D. W., “Antenna mechanism and deaggregation concept: novel mechanistic principles for photocatalysis," Comptes Rendus Chim. 9(5-6), 761-773 (2006).

[2] Li, Z., Liu, R.., Xu, Y., "Larger Effect of Sintering Temperature Than Particle Size on the Photocatalytic Activity of Anatase TiO2," J. Phys. Chem. C 117, 24360-24367 (2013).

[3] Sze., Physics of Semiconductor Devices, 3rd. ed., America (NY). 10(1), 3rd. ed., Wiley (2007).

[4] Ryu, J.., Choi, W., "Substrate-specific photocatalytic activities of $\mathrm{TiO} 2$ and multiactivity test for water treatment application," Environ. Sci. Technol. 42(1), 294-300 (2008).

[5] Kessler, R. W.., Wilkinson, F., "Diffuse Reflectance Triplet-Triplet Absorption Spectroscopy of Aromatic Hydrocarbons Chemisorbed on [Gamma]-Alumina," J. Chem. Soc., Faraday Trans. 1 77(2), 309-320 (1981).

[6] Schneider, J., Nikitin, K., Wark, M., Bahnemann, D. W.., Marschall, R., "Improved charge carrier separation in barium tantalate composites investigated by laser flash photolysis," Phys. Chem. Chem. Phys. 18, 10719-10726, Royal Society of Chemistry (2016).

[7] Schneider, J., Matsuoka, M., Takeuchi, M., Zhang, J., Horiuchi, Y., Anpo, M.., Bahnemann, D. W., "Understanding TiO2 Photocatalysis : Mechanisms and Materials," Chem. Rev. 114, 9919-9986 (2014). 